\title{
An experimental method to measure dynamic stress-strain relationship of materials at high strain rates
}

\author{
Xianqian $\mathrm{Wu}^{\mathrm{a}}{ }^{\mathrm{b}}$, Xi Wang ${ }^{\mathrm{a}}$, Yanpeng Wei ${ }^{\mathrm{a}}$, Hongwei Song ${ }^{\mathrm{a}}$, Chenguang Huang ${ }^{\mathrm{a}, *}$ \\ ${ }^{a}$ Key Laboratory of Mechanics in Fluid Solid Coupling Systems, Institute of Mechanics, Chinese Academy of Sciences, Beijing 100190, PR China \\ ${ }^{\mathrm{b}}$ Mechanical and Aerospace Engineering, Case Western Reserve University, Cleveland, OH 44106, USA
}

\section{A R T I C L E I N F O}

\section{Article history:}

Received 28 June 2013

Received in revised form

27 December 2013

Accepted 20 February 2014

Available online 13 March 2014

Keywords:

Dynamic behavior

Experimental method

Photonic Doppler velocimetry system

Polyvinylidene fluoride thin-film sensor

\begin{abstract}
A B S T R A C T
In this paper, an experimental method for measuring dynamic behavior of materials at high strain rates is developed. The strain and stress of a specimen are obtained using a high-precision photonic Doppler velocimetry system and a sensitive polyvinylidene fluoride thin-film sensor respectively. Based on the assumptions of one-dimensional stress state and negligible volume change at plastic deformation stage, the dynamic stress-strain relationship of materials can be determined. Using Al-2024T351 alloy and copper as model materials, the method demonstrates its effectiveness in measuring dynamic behavior of materials at high strain rates.
\end{abstract}

(ㄷ) 2014 Elsevier Ltd. All rights reserved.

\section{Introduction}

The understanding of dynamic behavior of materials plays an important role in many applications such as penetration, explosion and crashworthiness [1]. The split Hopkinson pressure bar (SHPB) technique, first introduced by Hopkinson [2] in 1914 and further developed by Kolsky [3], Davies and Hunter [4], is an effective experimental technique to determine the dynamic stress-strain relationship of materials at strain rates of $50 \mathrm{~s}^{-1}$ to $10^{4} \mathrm{~s}^{-1}$ [5-13]. The SHPB technique is based on the following assumptions: onedimensional wave propagation theory; uniform stress and strain distribution in axial direction; and negligible inertia effect $[3,4,11]$. To satisfy these assumptions, the lengths of the incident and transmitted bars can reach up to several meters especially for coarse grain heterogeneous materials [14]. Long bars inevitably introduce imperfect experimental conditions such as un-coaxiality and non-parallelism between bars, which will influence the stress state [15]. On the other hand, the measurement of SHPB technique is based on the wave propagation theory and the superposition principle [2-4]. The incident, reflected and transmitted waves are measured by strain gauges on the bars instead of at the specimen interfaces. The measured strains need to be shifted from the position of the strain gauges to the specimen in time and space. Errors

\footnotetext{
* Corresponding author. Tel.: +86 1082543879.

E-mail address: huangcg@imech.ac.cn (C. Huang).
}

might arise due to the dispersion effect, i.e. the change of the wave shape while propagating along the bar. Additionally, it is difficult to make an exact estimation of the shifting time to ensure the same beginning of the three waves $[4,11,16,17]$.

Many researchers have tried to improve the SHPB technique to broaden its application, including dispersion correction, strain field measurement and data processing methodology [8,11,12,14,16-23]. There are still some aspects needed to be improved. For instance, the digital image correlation (DIC) is one of the most popular methods for obtaining full-field deformation information. However, it is difficult for measuring the out-of-plane displacement, and the measurement of large in-plane displacement is also hard as the great decreasing of image contrast [22,23]. In this paper, we develop an experiment method to characterize high strain-rate behavior of materials. The strain is determined by the radial particle velocity of specimen measured with a high-precision velocimetry, and the stress is measured with a pressure sensor, respectively.

The paper is organized as follows. In Section 2, the measurement concept is described in detail. In Section 3, the experimental based on the method is established. In Section 4, we explain the measurement result and provide discussions relevant to this experimental method.

\section{Measurement concept}

The schematic diagram of the proposed experimental method is depicted as Fig. 1. The cylinder specimen is sandwiched between 


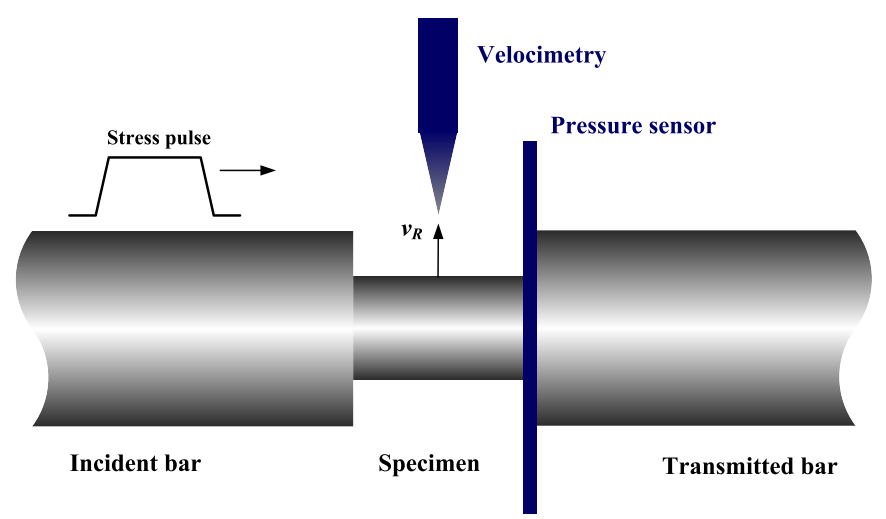

Fig. 1. Schematic diagram of the developed measurement method for stress-strain relationship at high strain rates.

two elastic rods denoted as incident bar and transmitted bar. While a stress pulse propagates through the specimen, the specimen experiences a dynamic compression deformation. The deformation of specimen causes a radial particle velocity at the specimen outside surface, which can be related to the longitudinal deformation of specimen through its deformation law. Therefore, it is possible to obtain the stress-strain response of material if we can measure the stress pulse and the radial velocity.

The proposed experimental method is based on two assumptions:

i. The stress in the specimen quickly reaches its equilibrium and is in one-dimensional state;

ii. The volume change at the plastic deformation stage is negligible.

The one-dimensional stress state is usually satisfied when the friction effect is negligible [2-4]. As pointed out by Davies and Hunter [24], the stress can reach an equilibrium state after the stress pulse experiences approximately $\pi$ reverberations within the specimen, i.e., the time for reaching an equilibrium state is approximately

$t_{\text {equil }}=\frac{\pi L_{0}}{c_{L}}$

where $L_{0}$ is length of specimen and $c_{L}$ is longitudinal wave speed in specimen. For a 5 -mm-thick specimen, taking $c_{L}=5000 \mathrm{~m} / \mathrm{s}$, the equilibrium time is expected to approximately $3 \mu \mathrm{s}$. Therefore, the specimen will deform uniformly quickly after the impact. At this stage, the stress at the back-surface where the pressure sensor is placed can be regarded as the stress at the position where the radial velocity is measured. According to Bridgman [25], the volume change at the plastic deformation stage is negligible for metals when the applied pressure is not very high. With this assumption, the longitudinal strain can be determined from the radial particle velocity through the deformation law.

The analysis of longitudinal strain can be divided into stages of elastic deformation and plastic deformation respectively. For the elastic deformation stage, the radial strain is related to the longitudinal strain owing to the Poisson's effect,

$\varepsilon_{r}=\frac{\partial u_{r}}{\partial r}=-\nu \varepsilon_{l}$

where $\varepsilon_{r}, \varepsilon_{l}$ denote radial strain and longitudinal strain respectively, $u_{r}$ is radial displacement, and $\nu$ is the Poisson's ratio.
Considering longitudinal strain $\varepsilon_{l}$ as a function of Lagrange coordinate $X$ and transient time $t, \varepsilon_{l}=\varepsilon_{l}(X, t)$, at the specimen surface ( $r=R, R$ is the radius of the cylinder specimen), the radial displacement $u_{R}$ can be expressed using the longitudinal strain $\varepsilon_{l}$ $(X, t)$ as

$u_{R}=-\nu R \varepsilon_{l}(X, t)$.

Consequently, the longitudinal strain $\varepsilon_{l}(X, t)$ can be solved by the radial particle velocity $v_{R}$,

$\varepsilon_{l}(t)=-\frac{1}{\nu R} \int_{0}^{t} v_{R}(t) d t$.

For the plastic deformation stage, the volume change is negligible. Assuming the longitudinal particle velocities at two positions with infinitesimal length $\Delta l$ of specimen are $v_{1}$ and $v_{2}$ respectively, the volume of the $\Delta l$-length specimen at time $t$ is

$V_{t}=\pi R_{t}^{2} \cdot \Delta l_{t}$

where $R_{t}$ and $\Delta l_{t}$ denote the radius and length of the $\Delta l$-length specimen at time $t$ respectively.

At time $t+\Delta t$, the volume of the $\Delta l$-length specimen is

$V_{t+\Delta t}=\pi R_{t+\Delta t}^{2} \cdot \Delta l_{t+\Delta t}=\pi\left[R_{t}+v_{R}(t) \cdot \Delta t\right]^{2} \cdot\left[\Delta l_{t}+\left(v_{2}-v_{1}\right) \cdot \Delta t\right]$.

Moreover, the strain rate of the specimen can be written as

$\dot{\varepsilon}_{l}(t)=\left(v_{2}-v_{1}\right) / \Delta l_{t}$.

Therefore, the relationship between the longitudinal strain and the radial particle velocity can be deduced as

$\varepsilon_{l}(t)=-\int_{0}^{t_{C}} \frac{v_{R}(t)}{\nu R} d t-\int_{t_{C}}^{t} \frac{2 v_{R}(t)}{R_{t}} d t$,

where $t_{C}$ is the critical time, at which the specimen transits from the elastic deformation to plastic deformation. As will be shown later, this critical time can be approximately obtained from the stress profile measured by the pressure sensor. The radius of the specimen at time $t, R_{t}$, is derived as

$R_{t}=R_{0}+\int_{0}^{t} v_{R}(t) d t$

From Eqs. (4) and (8), the longitudinal strain of the specimen can be calculated. Meanwhile, the related strain rate can also be determined,

$\dot{\varepsilon}_{l}(t)=\left|\frac{d \varepsilon_{l}(t)}{d t}\right|$.

Finally, the dynamic stress-strain relationship of the specimen can be obtained.

\section{Experimental}

\subsection{Experimental setup}

Even though a new experimental set-up can be established to validate the proposed method according to Fig. 1, the proposed experimental method is performed in a traditional SHPB 
experimental system consisted of a striker, an incident bar and a transmitted bar. The reason why we chose the traditional SHPB system to conduct the experiment is as follows. For the proposed method itself, we only need the SHPB system to provide a wellcalibrated stress pulse. Meanwhile, we can also obtain the stressstrain curve of the specimen using traditional strain gauges firmly cemented on the Hopkinson bar as an independent comparison baseline for the proposed method.

The geometry and material parameters for the SHPB system are listed in Table 1 [26]. The striker launched by the compressed nitrogen gas is used to generate an elastic stress pulse while it impacts the incident bar. When the incident stress wave arrives at the incident bar/specimen interface, a reflected wave is generated and propagates backwards along the incident bar due to the impedance mismatch effect, and the rest transmits through the specimen to the transmitted bar. During the experiment a laser velocimetry and a thin film pressure sensor are used to measure the outsider radial velocity and end-surface pressure of the specimen, respectively. As mentioned above, the strains in the incident and transmitted bars are also measured by the strain gauges.

Two types of well-studied metallic materials, Al-2024T351 alloy and copper, are tested in the experiments. Both materials are in the cylinder form. Al-2024T351 specimens have two kinds of dimensions, i.e. $\Phi 10 \times 10 \mathrm{~mm}$ and $\Phi 8 \times 8 \mathrm{~mm}$, while copper specimens have only one dimension, i.e. $\Phi 8 \times 8 \mathrm{~mm}$. The surfaces of those specimens are finely polished. A thin layer of lubricant is applied on the top and bottom surfaces of cylindrical specimen to minimize the friction between the bar and specimen.

A $25.0 \mu \mathrm{m}$ thick PVDF sensor coated with $12.5 \mu \mathrm{m}$ thick PTFE films is sandwiched between the specimen and the transmitted bar to record the stress of the specimen. The linear response of this PVDF thin-film sensor to transient pressures can reach up to several GPa $[27,28]$. It is competent for measuring pressure of hundreds of MPa which is the level of the yield strength of metallic materials. A current circuit, in which a $50 \Omega$ resistor is in parallel with the PVDF sensor, is used to record the electric charges discharged by the PVDF sensor.

A photonic Doppler velocimetry (PDV) system [15,29-32] is used to measure the radial particle velocity of the specimen as illustrated in Fig. 2. It consists of a cw laser with narrow linewidth, a fiber optic circulator, a high bandwidth detector, an optical collimating lens probe and an oscilloscope with high bandwidth and sample rate. The laser and the detector are connected onto the first and the third port of the circulator, respectively, and the probe is connected onto the second port of the circulator. When a laser illuminates the moving surface with a probe, a fraction of incident light is reflected at the interface between the probe lens and air. It acts as the reference light with the original laser frequency $f_{0}$. The signal light with frequency $f_{b}$ reflected from the measured surface is collected by the same probe. When the reference light and the signal light interfere in a coupler, the heterodyne frequency of the two lights, $f_{\text {het }}(t)$, is record by a high-bandwidth oscilloscope. The velocity of the measured surface can be determined,

$v(t)=\frac{\lambda_{0}}{2} f_{\text {het }}(t)$,

Table 1

Geometry and material parameters of the SHPB setup.

\begin{tabular}{llllll}
\hline $\begin{array}{l}\text { Length of Length of incident } \\
\text { striker } \\
(\mathrm{cm})\end{array}$ & $\begin{array}{l}\text { and transmitted } \\
\text { bar }(\mathrm{cm})\end{array}$ & $\begin{array}{l}\text { Diameters for Yong's } \\
\text { projectile and modulus } \\
\text { bars }(\mathrm{mm})\end{array}$ & $\begin{array}{l}\text { Initial density Poisson's } \\
\rho\left(\mathrm{Gg} / \mathrm{m}^{3}\right)\end{array}$ & $\begin{array}{l}\text { Patio } \nu \\
\text { ration }\end{array}$ \\
\hline 30 & 120 & 16 & 206 & $7.85 \times 10^{3}$ & 0.3 \\
\hline
\end{tabular}

where $\lambda_{0}$ is the original wavelength of the laser. The Morlet wave analysis method is used to get the velocity profile according to the heterodyne frequency $f_{\text {het }}(\mathrm{t})$. The data processing method is given in detail in our previous work [22].

A CQF938 series $40 \mathrm{~mW}$ operating at $1550 \mathrm{~nm}$ continuous wave (CW) distributed feedback (DFB) laser with a polarization maintaining fiber (PMF) and a narrow line-width of about $200 \mathrm{kHz}$ is used. An InGaAs PIN photodiode detector (New Focus) with a bandwidth of $12 \mathrm{GHz}$ is employed. A Lecory WaveMaster 808Zi oscilloscope with a bandwidth of $8 \mathrm{GHz}$ and a sampling rate of $40 \mathrm{GS} / \mathrm{s}$ is adopted to record the interference signal of the PDV system and the voltage charged by the PVDF sensor. The probe with a diameter of about $0.3 \mathrm{~mm}$ is equipped with a $15 \mathrm{~mm}$ focuser. In the experiments, the probe of the PDV system is placed opposite to the specimen surface and aligned to the radial direction in order to capture the radial particle velocity precisely.

As the strains are also measured through the strain gauges attached on the bars, the stress-strain relationships of specimen can also be derived from the traditional SHPB theory. Denote the incident strain $\varepsilon_{I}(t)$ and reflective strain $\varepsilon_{R}(t)$ in the incident bar and transmitted strain $\varepsilon_{T}(t)$ in the transmitted bar, the stress and strain in the specimen can be obtained as follows:

$$
\begin{aligned}
& \sigma_{S}(t)=E \frac{A_{0}}{A_{S}} \varepsilon_{T}(t) \\
& \dot{\varepsilon}_{S}(t)=-2 \frac{c_{0}}{L_{S}} \varepsilon_{R}(t) \\
& \varepsilon_{S}(t)=\int_{0}^{t} \dot{\varepsilon}_{S}(t) d t
\end{aligned}
$$

In Eqs. (12)-(14), $E, A_{0}$ and $c_{0}$ are Young's modulus, crosssectional area, and longitudinal wave speed in incident and transmitted bars, respectively, and $A_{s}$ and $L_{s}$ are the initial cross-sectional area and length of the specimen, respectively. The true stressstrain relationship is determined from the engineering stressstrain relationship by assuming uniform and isochoric deformation conditions to prevail within the specimen during the deformation process.

\subsection{Calibration}

The accuracy of the stress-strain relationship determined with the proposed method depends on the precision of the PVDF sensor and the PDV system and needs to be investigated. Firstly, the compressive piezoelectric coefficient $K$ of the PVDF sensor is calibrated using the same SHPB apparatus as described in the previous section. The PVDF sensor is directly sandwiched between the incident bar and the transmitted bar. A thin layer of lubricant is used at both side of the thin film to minimize friction between the bars and PVDF sensor. The stress pulse of transmitted bar, which is measured by strain gauge, is used to determine the coefficient $K$ as follows,

$K(t)=\frac{\int_{0}^{t}\left[U_{R}(t) / 50\right] d t}{E \varepsilon_{T}(t) \cdot A}$,

where $U_{R}(t)$ is the voltage charged by PVDF sensor, $E, \varepsilon_{T}(t)$ and $A$ are the Young's modulus, strain and cross-section area of the transmitted bar. The measured results for $U_{R}(t)$ and $\varepsilon_{T}(t)$ are shown in Fig. 3. The fast increase and decrease of voltage indicate the fast rise and fall stage of stress pulse. The rise-time duration and the full 


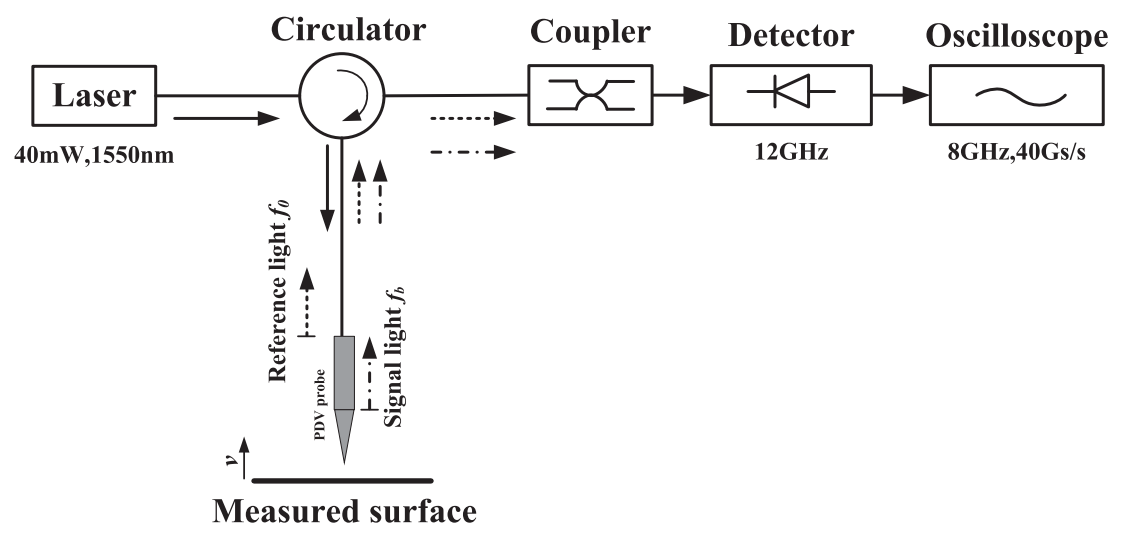

Fig. 2. Configuration of the PDV system.

duration of the voltage pulse agree with the stress pulse of transmitted bar. The plateau stages of the integrated voltage signal and the transmitted stress signal are taken in the calibration experiments, and the coefficient $K(t)$ is almost constant during the plateau stage. Totally five tests are performed, and the calculated coefficients $K$ are shown in Fig. 4, from which the coefficient $K$ of the PVDF pressure sensor is determined as $24.2 \pm 0.89 \mathrm{pC} / \mathrm{N}$. Although the maximum error of the velocity measured by the PDV system is expected less than 0.1\% [29], this developed PDV system is also calibrated on this SHPB apparatus. Only the projectile and the incident bar are used. The projectile is launched using the compressed gas and impacts the incident bar. The launching velocity of the projectile is measured with three photodiode array. When the projectile reaches a photodiode, an electrical signal is generated. The velocity of the projectile is calculated from those electrical signals of the photodiode array. While the projectile impact the incident bar, the central longitudinal velocity of the free surface of the incident bar is measured by the PDV system. The measured raw data and the velocity profile are given in Fig. 5. The density of the raw infringe data indicates the velocity amplitude. The closer the two adjacent fringes are, the faster the velocity at the moment is. The fast rising edge and the fluctuation of dispersion effect induced by lateral inertia of the velocity are captured. The end-surface velocity of the incident bar measured by the PDV system are about $19.80 \mathrm{~m} / \mathrm{s}$ in average, which is consistent with the measurement of about $19.87 \mathrm{~m} / \mathrm{s}$ from the photodiode array.

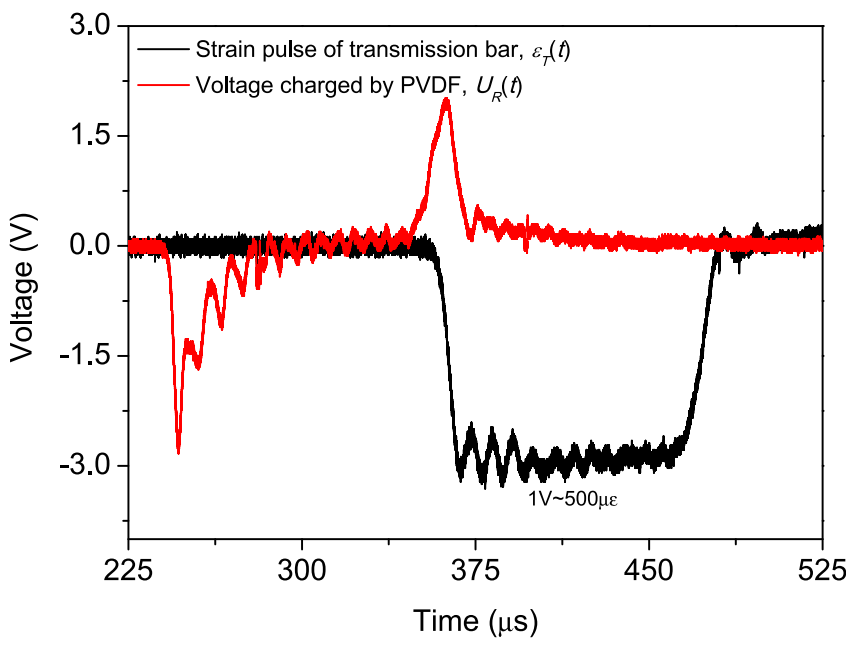

Fig. 3. Voltage charged by PVDF pressure sensor and strain pulse of transmitted bar.

\section{Results and discussion}

The experiment was performed to measure the stress-strain relationship of materials at high strain rates. The typical results for Al-2024T351 alloy are shown in Figs. 6-8 to demonstrate the proposed method. The specimen is placed coaxially with the bars. The velocity of the projectile measured by the photodiode array is $12.52 \mathrm{~m} / \mathrm{s}$. The measurement of the PVDF sensor is triggered simultaneously with the measurement of the PDV system. A typical result of voltage measured by the PVDF sensor and the corresponding stress is shown in Fig. 6. The stress is calculated by integrating the PVDF signal. The two peaks in the initial stage of PVDF signal is due to the slightly change of the stress' slop. The stress measured by the PVDF sensor indicates the dynamic behavior of the specimen. At about $2 \mu \mathrm{s}$, the stress wave reaches the interface between the specimen and the PVDF sensor. The stress increases linearly and quickly up to about $406 \mathrm{MPa}$ at about $12.7 \mu \mathrm{s}$, which means the elastic deformation stage for the specimen. Then it increases relative slowly with slight fluctuation for about $20 \mu \mathrm{s}$ duration, which indicates the specimen begin to yield. Based on the stress measurement result, the duration of the elastic deformation stage is about $10.7 \mu$ s. Therefore, the critical time at which the elastic deformation stage ends, $t_{C}$, is determined as about $10.7 \mu$ s for the analysis of the strain according to the radial particle velocity measured by the PDV system. After that, the stress increases linearly and much slowly to about $527 \mathrm{MPa}$ followed with an unloading

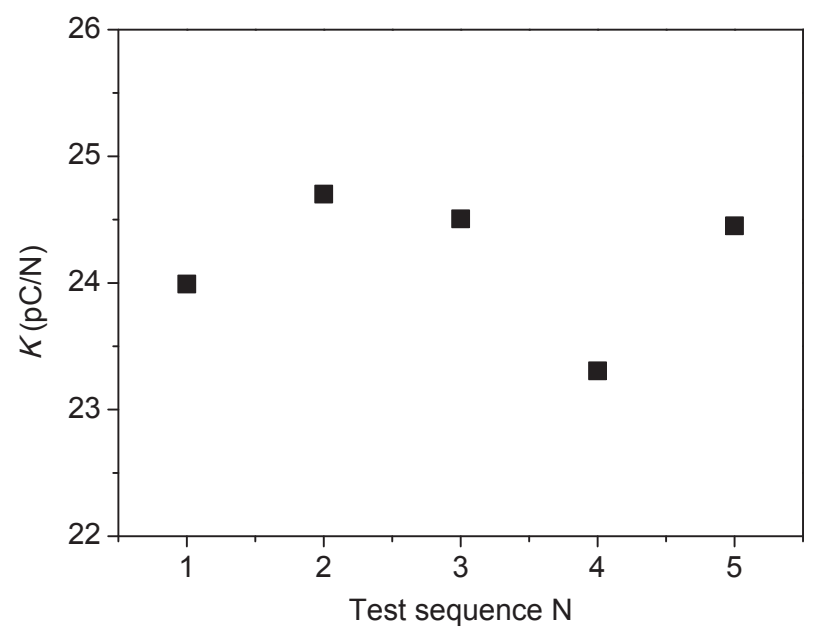

Fig. 4. Calculated compressive piezoelectric coefficients of the PVDF sensor. 


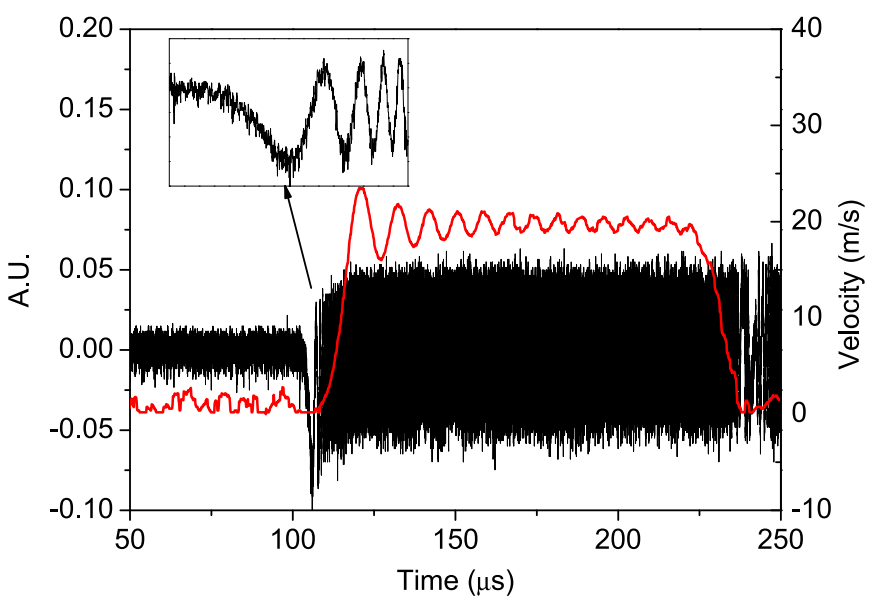

Fig. 5. Raw data and the corresponding velocity profile measured by the PDV system.

process. As the maximum pressure applied on the specimen is less than $600 \mathrm{MPa}$, the volume change can be negligible [25]. Fig. 7 shows the radial particle velocity at the middle position of the specimen measured by the PDV system, in which the dynamic response of the specimen is also revealed. At the beginning, the

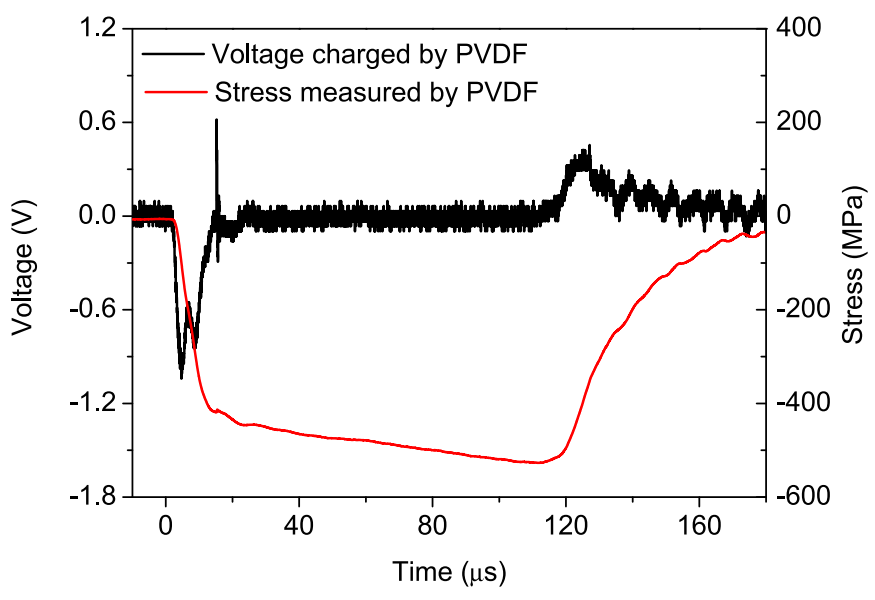

Fig. 6. Voltage and related stress measured by the PVDF sensor.

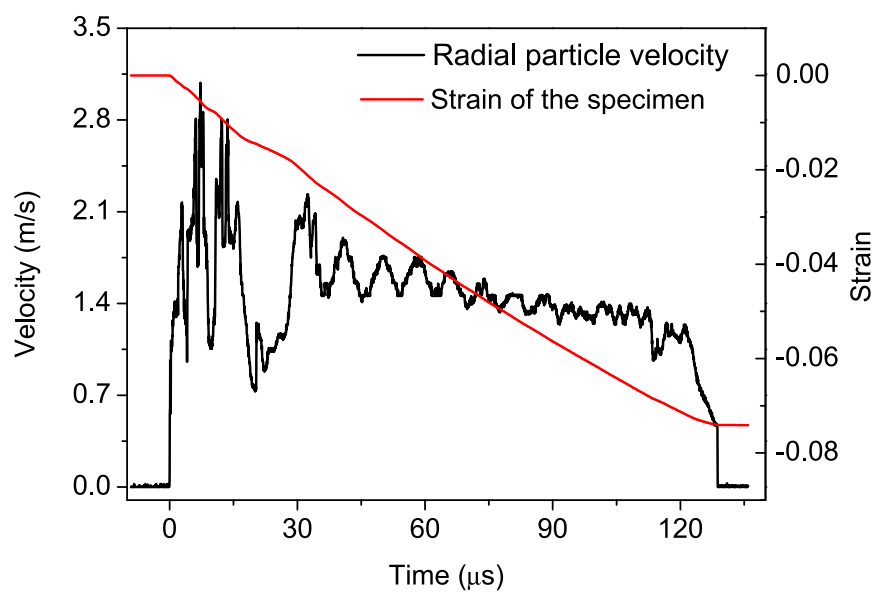

Fig. 7. Radial particle velocity and related longitudinal strain of the specimen measured by the PDV system.

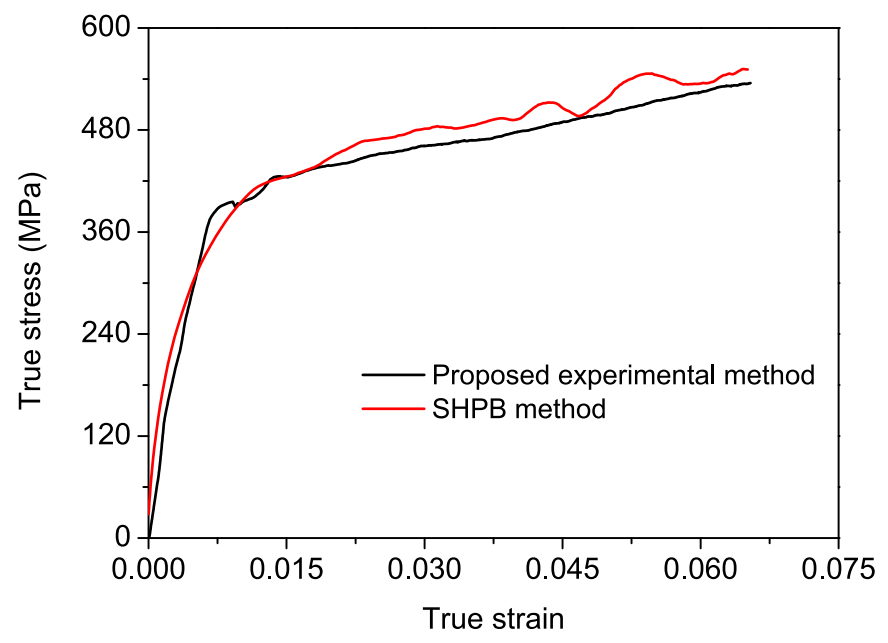

Fig. 8. True stress-strain curves measured by the developed experimental method and the SHPB method.

radial particle velocity of the specimen changes fast with a relative high amplitude of about $2.8 \mathrm{~m} / \mathrm{s}$ for nearly $12 \mu \mathrm{s}$ duration. After a relatively low value of $1 \mathrm{~m} / \mathrm{s}$ for a period of about $10 \mu \mathrm{s}$, the radial particle velocity decreases gradually until the end of loading. As the probe of the PDV system placed at the middle position of the specimen, the start time of the radial particle velocity is about $2 \mu \mathrm{s}$ earlier than that of the stress measurement result. The theoretical transfer time for the stress waves in that length is about $1 \mu \mathrm{s}$. A relative large value (about $2 \mu \mathrm{s}$ ) is measured in the experiment, which is ascribed to the imprecision selection on the start time of the velocity and the pressure. However, the error introduced by the small difference of the time shifting could be negligible. Note the measurement of the PDV system is the Euler velocity $v_{E}$ rather than the Lagrange velocity $v_{L}$. The Lagrange velocity $v_{L}$ can be related to the Euler velocity $v_{E}$ as $v_{L}=v_{E} /(1+\varepsilon)$, where $\varepsilon$ is the longitudinal strain. Based on the PDV measurement result, the longitudinal strain is determined using Eqs. (4) and (8) and is shown in Fig. 7. Note that a precise knowledge of the Poisson ratio is necessary in calculating the longitudinal strain from the radial velocity. A $1.0 \%$ error of the Poisson ratio will introduces $0.99 \%$ error of the strain value. However, if we suppose the volume change is also negligible during elastic deformation stage, the Poisson ratio would be not needed in the analysis, which will be investigated in the future. The strain rate of the specimen is also calculated as about $900 \mathrm{~s}^{-1}$ by Eq. (10). The true stress-strain relationship of Al-2024T351 material measured using the proposed method is shown in Fig. 8, where the engineering stress measured by the PVDF sensor is converted to the true stress. The true stress-strain relationship can be divided into two linear sections: elasticity and plasticity. The results show that the Young's modulus $E$ is about $74.7 \mathrm{GPa}$, the tangent modulus $E_{T}$ is about $1.63 \mathrm{GPa}$ and the yield stress $\sigma_{Y}$ is about $419 \mathrm{MPa}$. The results are consistent with the values reported in the literature [18,33,34]. As a comparison, the dynamic behavior of Al-2024T351 material was also measured using traditional SHPB technique with strain gauges. The measured strain pulses in the incident bar and the transmitted bar are shown in Fig. 9, from which the true stressstrain curve is determined with two-wave method as Eqs. (12)(14) and also depicted in Fig. 8. There is an agreement between these two curves from different method. More tests for $\Phi 8 \times 8 \mathrm{~mm}$ Al-2024T351 cylindrical specimens and $\Phi 8 \times 8 \mathrm{~mm}$ copper cylindrical specimens at different strain rate are performed with the method. The measurement results given in Fig. 10 are agree with the reported values [30-32], which shows the reliability of this 


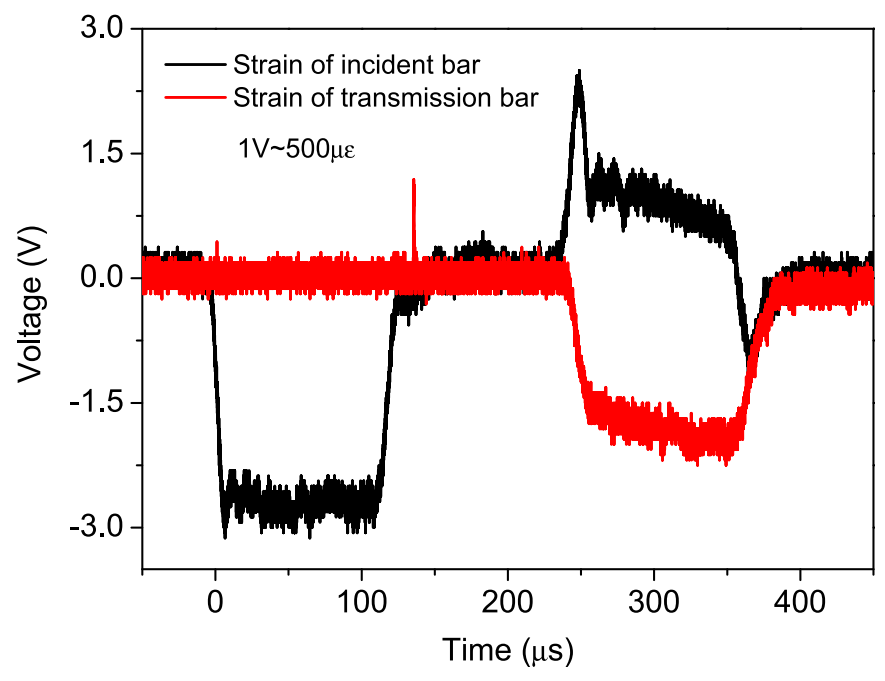

Fig. 9. Strain pulses of the incident bar and the transmitted bar of SHPB.

experimental method for measuring dynamic stress-strain relationship of metals.

To further validate this experimental method, a 3D numerical simulation is performed. A quarter symmetric model based on the experimental setup is built. The LS-DYNA [35] explicit nonlinear structural integration scheme is used to analyze the dynamic response of the Al-2024T351 cylindrical specimen especially the radial velocity and the stress at the back-surface. The striker is meshed with 48,000 eight nodes brick elements, and the incident bar and the transmitted bar are meshed with 192,000 eight nodes brick elements, respectively. The striker, the incident bar and the transmitted bar are finely meshed with 192 elements at the cross section. The specimen is finely meshed with 32,000 eight nodes brick elements, and its cross section is meshed with 640 elements. The elastic constitutive model is used for the striker, the incident bar and the transmitted bar. The material properties are given in Table 1 . The bilinear isotropic hardening constitutive model is used for the specimen. The material properties of the specimen are determined from the experimental result in Fig. 8. The weighted master-slave surface algorithm without considering friction [35] is chosen to model the contact behavior of interfaces. The simulated particle radial velocity at the middle position of the specimen is

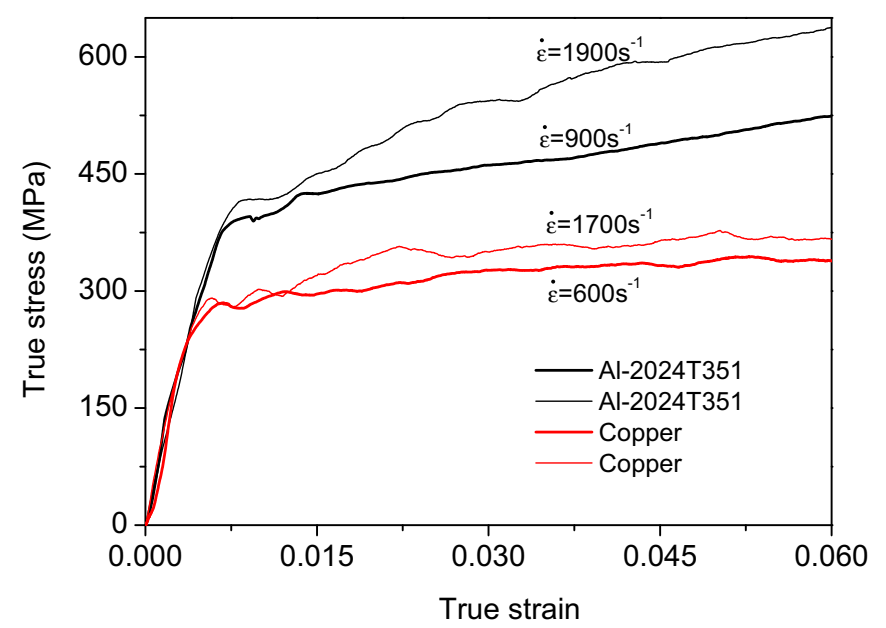

Fig. 10. True stress-strain curves of Al-2024T351 alloy and copper at different strain rate measured by the experimental method. shown in Fig. 11 and compared with the experimental result. There are some differences at the initial stage (region $\mathrm{A}$ ) and the end stage (region $C$ ) between the experimental measurement and the numerical simulation. The fast increase of the experimentally measured velocity during the short duration $A$ is not true, which could be ascribed to the data processing method for the PDV system. However, in Fig. 12 it will be seen that this difference at the duration A almost does not affect the strain history. The difference in the region $\mathrm{C}$ is also ascribed to the velocity measurement method. The simulation shows that the radial velocity changes its sign in region $C$, which means the material starts to elastic unload at this stage. Our PDV system can only detect the amplitude of the velocity of a moving surface but not its moving direction. Therefore the velocity measurement in region $C$ is not real. A PDV system with capability of distinguishing the sign of the velocity is under developing for the future experiment. The comparisons of the strain history and the back-surface stress of the specimen between the experimental measurement and the numerical simulation are given in Figs. 12 and 13. As the stress-strain curve used in the numerical simulation is not strictly same as the measurement result, the simulated strain and stress histories have very slight differences with the experimental measurements. Nevertheless, the simulated strain and stress histories are in agreement with the experimental measurements, which validates this experimental method for measuring materials' dynamic stress-strain relation ships.

As the strain and stress of the specimen are measured directly, errors induced by dispersion effects or imperfect experimental conditions could be minimized, and the out-of-plane displacement of specimen can be measured even for large deformation. The sensitivity of this method will be further investigated for different materials in the future. In addition, in SHPB the incident and reflected stress pulse should be separated, generally leading to long dimension of incident rod. For example, for a stress pulse with time duration $t$, the incident bar should be longer than $L_{\min }=c t$, where $c$ is the longitudinal elastic wave speed. Based on the proposed measurement method, the separation of the incident and reflected waves is not required. To obtain the same loading duration as SHPB, the incident bar should be longer than $L_{\min }=c t / 2$, which is the half of the length used in SHPB. This method could be useful especially for large size specimen such as concrete to avoid very long bars.

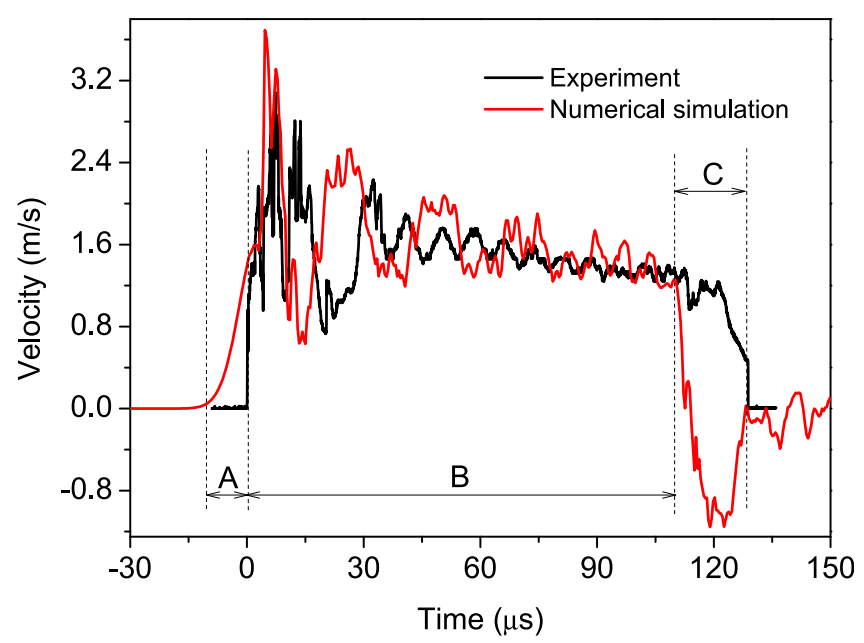

Fig. 11. Particle radial velocities at the middle of the specimen. The measured velocity profile at duration $A$ and duration $C$ are not true, which are ascribed to the velocity measurement system. 


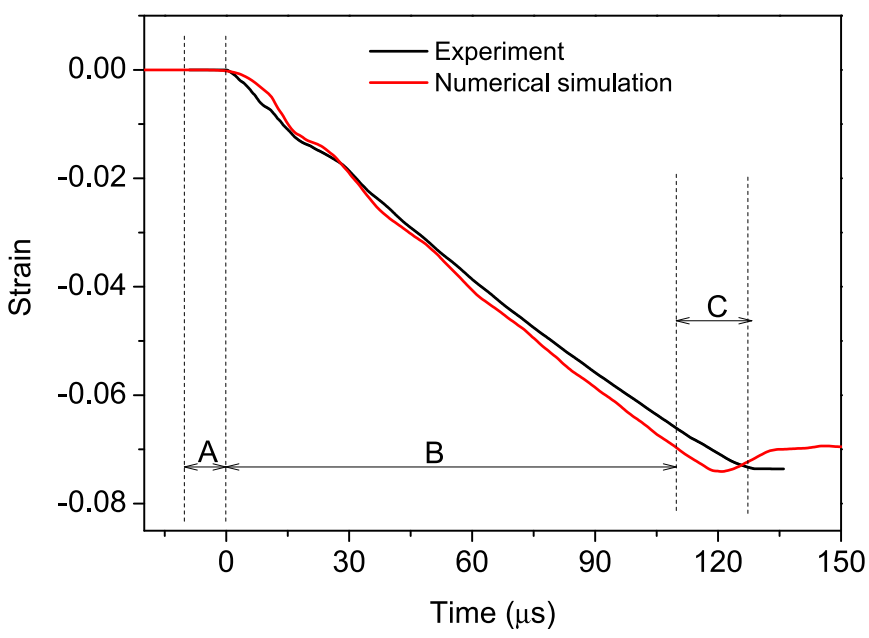

Fig. 12. Comparison of the strain histories of the specimen between the experimental measurement and the numerical simulation.

In the experiment, the effects of friction are neglected. Actually, the friction affects the one-dimensional stress state in the specimen, which will cause a difference of the stresses between the PDV measured position and the PVDF sensor measured position. In addition, the frictions between the specimen and the PVDF sensor leads to the generation of shear stress, which will contribute to the voltage charged by the PVDF sensor [28] and thereby decrease the accuracy of measured stress pulse. Thus, the frictions of interfaces should be diminished. The friction effects will be investigated in the future. Additionally, this method might also be useful to characterize the dynamic behavior of materials especially for the materials with relative low impedance. As the slow stress wave velocities in those materials, it is usually difficult to avoid the superposition of stress waves using the traditional SHPB technique. The direct measurements of strain and stress using this experimental method might be helpful. However, the volume change at plastic deformation stage should be taken into account for the measurements of these materials, and the experimental technique and related analysis method need to be improved to accommodate the volume change of a specimen at the plastic deformation stage. Moreover, this method is still at its early stage of development. Further improvement and investigation will be performed to enhance its applicability.

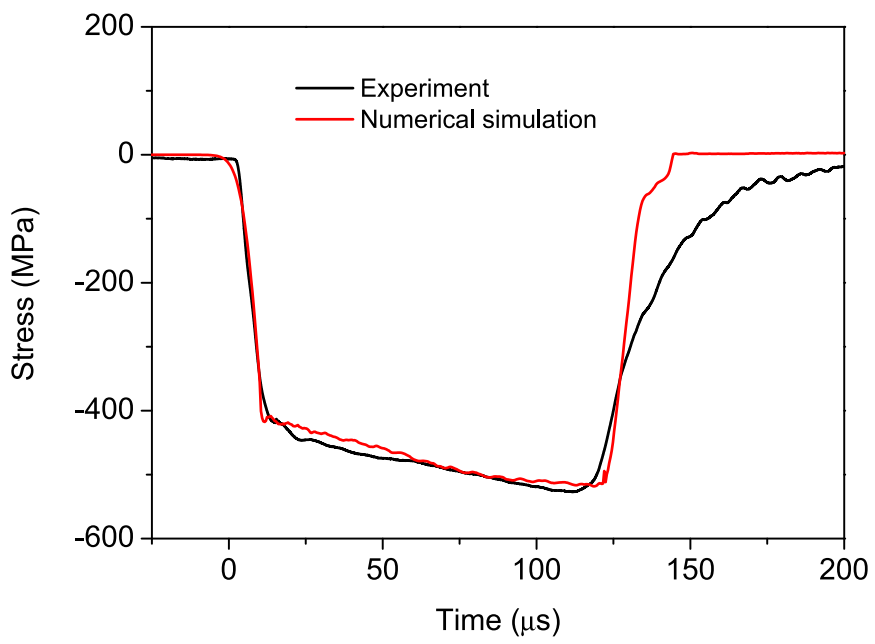

Fig. 13. Comparison of the end-surface stress histories of the specimen between the experimental measurement and the numerical simulation.

\section{Summary}

In this paper, we develop an experiment method to characterize the dynamic behavior of metals. The longitudinal strain of materials is calculated by the radial particle velocity measured by a PDV system, and the uniaxial stress is measured directly with a PVDF thin-film sensor. Using Al-2024T351 and copper as model materials, this method demonstrates some of its effectiveness and advantages. The errors introduced by the dispersion effects could be minimized, and the experimental set-up could be designed much compactly to decrease the influence of some imperfect experimental conditions. More tests with some materials where there are difficulties with SHPB such as concrete will be conducted in the future.

\section{Acknowledgments}

The authors would like to thank the National Natural Science Foundation of China (Grant No. 11332011) for financial support.

\section{References}

[1] Meyers M. Dynamic behavior of materials. 1st ed. New York: Wiley-Interscience; 1994. pp. 1-4.

[2] Hopkinson B. A method of measuring the pressure produced in the detonation of high explosives or by the impact of bullets. Philos Trans R Soc Lond A 1914;213:437-56

[3] Kolsky H. An investigation of the mechanical properties of materials at very high rates of loading. Proc R Soc Lond B 1949:62:676-700.

[4] Davies R. A critical study of the Hopkinson pressure bar. Philos Trans R Soc Lond A 1948;240:375-457.

[5] Bailly P, Delvare F, Vial J, Hanus JL, Biessy M, Picart D. Dynamic behavior of an aggregate material at simultaneous high pressure and strain rate: SHPB triaxial tests. Int J Impact Eng 2011;38:73-84.

[6] Gama BA, Lopatnikov SL, Gillespie Jr JW. Hopkinson bar experimental technique: a critical review. Appl Mech Rev 2004:57:223-50.

[7] Li Y, Guo Y, Hu H, Wei Q. A critical assessment of high-temperature dynamic mechanical testing of metals. Int J Impact Eng 2009;36:177-84.

[8] Li Z, Lambros J. Determination of the dynamic response of brittle composites by the use of the split Hopkinson pressure bar. Comp Sci Tech 1999;59:1097-107.

[9] Meng H, Li Q. Correlation between the accuracy of a SHPB test and the stress uniformity based on numerical experiments. Int I Impact Eng 2003;28:537-55

[10] Nemat-Nasser S. Introduction to high strain rate testing. In: ASM handbook, vol. 8. OH: ASM International; 2000. pp. 427-8.

[11] Zhao H. Material behaviour characterisation using SHPB techniques, tests and simulations. Comp Struct 2003;81:1301-10.

[12] Zhao H, Abdennadher S, Othman R. An experimental study of square tube crushing under impact loading using a modified large scale SHPB. Int J Impact Eng 2006;32:1174-89.

[13] Zhu J, Hu S, Wang L. An analysis of stress uniformity for concrete-like specimens during SHPB tests. Int J Impact Eng 2009;36:61-72.

[14] Merle R, Zhao H. On the errors associated with the use of large diameter SHPB, correction for radially non-uniform distribution of stress and particle velocity in SHPB testing. Int J Impact Eng 2006;32:1964-80.

[15] Wu X, Huang C, Wang X, Song $\mathrm{H}$. A new effective method to estimate the effect of laser shock peening. Int J Impact Eng 2011;38:322-9.

[16] Follansbee P, Frantz C. Wave propagation in the split Hopkinson pressure bar. J Eng Mat Tech 1983;105:61-6.

[17] Yew E, Chen C. Experimental study of dispersive waves in beam and rod using FFT. J Appl Mech 1978;45:940-3.

[18] Avinadav C, Ashuach Y, Kreif R. Interferometry-based Kolsky bar apparatus. Rev Sci Instrum 2011:82:073908.

[19] Gong J, Malvern L, Jenkins D. Dispersion investigation in the split Hopkinson pressure bar. J Eng Mater Technol 1990;112:309.

[20] Lennon A, Ramesh K. A technique for measuring the dynamic behavior of materials at high temperatures. Int J Plast 1998;14:1279-92.

[21] Lifshitz J, Leber H. Data processing in the split Hopkinson pressure bar tests. Int J Impact Eng 1994;15:723-33.

[22] Song H, Wu X, Huang C, Wei Y, Wang X. Measurement of fast-changing low velocities by photonic Doppler velocimetry. Rev Sci Instrum 2012;83: 073301-7.

[23] Peyre P, Scherpereel X, Berthe L, Fabbro R. Current trends in laser shock processing. Surf Eng 1998;14:377-80.

[24] Davies E, Hunter S. The dynamic compression testing of solids by the method of the split Hopkinson pressure bar. J Mech Phys Solids 1963:11:155-79. 
[25] Bridgman PW. Effects of high hydrostatic pressure on the plastic properties of metals. Rev Mod Phys 1945;17:3-14.

[26] Chen G, Chen Z, Tao J, Niu W, Zhang Q, Huang X. Investigation and validation on plastic constitutive parameters of 45 steel. Explo Shock Wave 2005;25: 451-6.

[27] Bauer F, Graham RA, Lee LM. Response of Bauer piezoelectric polymer stress gauges to shock loading. 1st ed. North Holland: Elsevier Science Publisher; 1987.

[28] Couturier S, de Ressguier T, Hallouin M, Romain J, Bauer F. Shock profile induced by short laser pulses. J Appl Phys 1996;79:9338.

[29] Dolan DH. Accuracy and precision in photonic Doppler velocimetry. Rev Sci Instrum 2010;81:053905-12.
[30] Dolan DH, Jones SC. Push-pull analysis of photonic Doppler velocimetry measurements. Rev Sci Instrum 2007;78:076102-5.

[31] Strand OT, Goosman DR, Martinez C, Whitworth TL, Kuhlow WW. Compact system for high-speed velocimetry using heterodyne techniques. Rev Sci Instrum 2006;77:083108-16.

[32] Wu X, Duan Z, Song H, Wei Y, Wang X, Huang C. Shock pressure induced by glass-confined laser shock peening: experiments, modeling and simulation. J Appl Phys 2011;110:053112.

[33] Bauccio M. ASM metals reference book. 3rd ed. OH: ASM International; 1993.

[34] Boyer HE, Gall TL. Metals handbook. OH: ASM International; 1985. pp. 414-7.

[35] Manual L.-D.K.U.s., version 970. Livermore, CA, USA: Livermore Software Technology Corporation; 2003. pp. 1-46. 